\title{
Estudos In silico sobre as atividades anticancerígenas do Eugenol presente no Cravo Da Índia (Syzygium aromaticum)
}

\author{
In silico Studies on the anticancerigenic activities of Eugenol in the Indian Carnation (Syzygium \\ aromaticum)
}

En Estudios In silico sobre las actividades anticancerigénicas del Eugenol en el Clavel Indio (Syzygium aromaticum)

\section{Resumo}

Objetivo: Investigar as propriedades anticancerígenas do eugenol usando métodos In silico. Metodologia: é um artigo original utilizando a metodologia In silico atraves dos softwares PASS (Prediction of activity spectra for substances) online e GUSAR disponíveis de forma gratuita e livre. Resultados: Os resultados do sistema operacional online PASS demonstraram diversas atividades biológicas positiva, tais quais efeito antimutagênico, estimulante de caspase, tratamento pré neoplásico e agonista de apoptose tendo uma probabilidade de atividade (PA) bem superior a 0,700. Tais achados reforçam aos descritos na literatura que evidenciam um potencial antineoplásico devido o componente ter efeitos antioxidante, e estar vinculado a expressão de genes envolvidos na apoptose e inflamação. Tal como efeitos na regulação negativa de Bcl-2, COX-2 e IL-1ß, e ações na regulação positiva de P53, Bax e expressão ativa de Caspase-3, caspase-9 além de induzir a via da apoptose mitocondrial. Já pelo software GUSAR, foi observado a toxicidade, demonstrando que na categoria 4, estão as vias de administração intravenosa, oral, e subcutânea, indicando que é pouco tóxico. Já na categoria 5 enquadra-se a via intraperitoneal é relativamente atóxico. Tal achado ainda é obscuro e possui poucos artigos científicos para o embasamento. Conclusão: O Eugenol possui efeitos favoráveis para o tratamento e prevenção de diversos canceres e células mutagênicas, porém ainda há pouco conhecimento sobre a toxicidade deste componente. Assim, necessita de um maior aprofundamento sobre a temática.

Palavras-chave: Eugenol; Anticancerígeno; Antimutagênico; In silico.

\begin{abstract}
Objective: To investigate the anti-cancer properties of eugenol using In Silico methods. Methodology: it is an original article using the In silico methodology through PASS (Prediction of activity spectra for plants) software online and GUSAR available for free and acessful. Results: The results of the online PASS operating system demonstrated several positive biological activities, such as antimutagenic effect, caspase stimulant, pre-neoplasic treatment and apoptosis agonist having a probability of activity (BP) well above 0.700 . Such findings reinforce the others in the literature that show an antineoplastic potential because the component has antioxidant effects, and is linked to an expression of genes involved in apoptosis and inflammation. Such as effects on the negative regulation of Bcl-2, COX-2 and IL-1ß, and actions on the positive regulation of P53, Bax and active expression of Caspase-3, caspase-9 in addition to inducing the pathway of mitochondrial apoptosis. As for the GUSAR software, toxicity was observed, demonstrating that in category 4, they are intravenous, oral, and subcutaneous routes of administration, indicating that it is not very toxic. In category 5 , the intraperitoneal route is relatively non-toxic. This finding is still obscure and there are few scientific articles to support it. Conclusion: Eugenol has favorable effects for the treatment and prevention of various cancers and mutagenic cells, but there is still little knowledge about the toxicity of this component. Thus, it needs to go deeper into the theme.
\end{abstract}

Keywords: Eugenol; Anticancerigen; Antimutagenic; In silico.

\section{Resumen}

Objetivo: Investigar las propiedades anticancerígenas del eugenol utilizando métodos In silico. Metodología: se trata de un artículo original utilizando la metodología In silico a través del software PASS (Predicción de espectros de 
actividad para sustancias) online y GUSAR disponible de forma gratuita y accesible. Resultados: Los resultados de los sistemas operativos PASS en línea demostraron varias actividades biológicas positivas, como efecto antimutagénico, estimulante de caspasa, tratamiento preneoplásico y agonista de apoptosis con una probabilidad de actividad (PA) muy superior a 0,700. Dichos hallazgos refuerzan los descritos en la literatura, que muestran un potencial antineoplásico debido a que el componente tiene efectos antioxidantes y está ligado a la expresión de genes involucrados en la apoptosis y la inflamación. Tales como efectos sobre la regulación negativa de Bcl-2, COX-2 e IL$1 \beta$, y acciones sobre la regulación positiva de P53, Bax y expresión activa de Caspasa-3, caspasa-9 además de inducir la vía de apoptosis mitocondrial. En cuanto al software GUSAR, se observó toxicidad, demostrando que en la categoría 4, se encuentran las vías de administración intravenosa, oral y subcutánea, lo que indica que no es muy tóxico. En la categoría 5, la vía intraperitoneal es relativamente no tóxica. Este hallazgo aún es oscuro y hay pocos artículos científicos que lo respalden. Conclusión: El eugenol tiene efectos favorables para el tratamiento y prevención de varios cánceres y células mutagénicas, pero aún hay poco conocimiento sobre la toxicidad de este componente. Por tanto, es necesario profundizar en el tema.

Palabras clave: Eugenol; Anticancerígeno; Antimutagénico; In silico.

\section{Introdução}

O cravo da índia (Syzygium aromaticum) é uma especiaria pertencente à família Myrtacea originada da Ilha Molucas, localizado na Indonésia (Affonso, Rennó, Slana \& Franca, 2012)

Diversos estudos fitoquímicos realizados com o cravo da índia mostram uma grande proporção de óleo essencial, chegando até $90 \%$, onde o eugenol é o componente majoritário, seguido pelos compostos trans-cariofileno, acetato de eugenila e a-humuleno (Paoli et al., 2007; Pereira et al., 2008).

Entre as atividades biológicas reveladas do eugenol, destacam-se as propriedades antibacteriana, antifúngica, antioxidante, anti-inflamatória, anestésica, antiviral, antihelmintos e anticancerígenas (Roque, 2014; Scherer, Wagner, Duarte, Godoy, 2009).

No Brasil, esta especiaria chegou em 1770. Contudo, hoje está entre o $3^{\circ}$ maior produtor do cravo-da-índia, tendo uma colheita de 6 mil toneladas /ano (Lutfi, Roque, 2014).

O eugenol mostrar-se como um líquido incolor, amarelo claro ou âmbar. Apresenta odor de cravo e gosto picante (NTP, 1992). Em relação as suas propriedades anticancerígenos, estudos tem apontado que eugenol previne a progressão do câncer por meio da modulação da expressão de vários genes envolvidos no crescimento celular, angiogênese e apoptose (AlSharif et al, 2013). Em um modelo de carcinogênese gástrica em ratos, realizado por Manikandan el al (2010), foi observado que o eugenol induz a apoptose e inibe a invasão e a angiogênese.

A previsibilidade computacional da atividade transige a beneficência de filtrar componentes que seriam considerados vagamente promissores em fases ainda iniciais de uma pesquisa. As novas tendências são consideradas para o uso do de metodologias In silico (Lutfi \& Roque, 2014).

In silico é uma denotação para o uso de simulações computacionais que modelam um processo natural ou de laboratório. Assim, tais programas utilizam do cruzamento de informações contidas em banco de dados e das formulações e estruturas químicas das substancias para preverem as propriedades, atividades biológicas, ações toxidades, ou quaisquer outras funções. (Filimonov et al, 2014).

A utilização desta técnica foi Descrita pela primeira vez em 1989 no workshop "Autômatos Celulares: Teoria e Aplicações", em Los Alamos (EUA) (Sotomayor \& Schulten ,2007). Assim, essa ferramenta permite: otimizar a triagem, testando apenas os compostos mais promissores; diminuir gastos, recursos, uso de animais; prever propriedades sobre a dose media letal; entre outros benefícios. Contudo, ainda há algumas barreiras do uso de seu método, tais como: o conhecimento limitado por parte os profissionais e acadêmicos, a maioria dos estudos estão escritos em língua estrangeira, dificultando o acesso a muitos. E uma gama de programas excepcionais não são gratuitos, limitando o acesso de tal ferramenta (Danchin, Medigue, Gascuel, Soldano \& Henaut, 1991; Palsson, 2000). 
Dessa foram, o objetivo desse estudo foi investigar as propriedades anticancerígenas e toxicológicas do eugenol usando softwares gratuitos.

\section{Metodologia}

Trata-se de um estudo In silico, quantitativo. A pesquisa quantitativa caracteriza-se pela utilização de dados coletados por meio do uso de medições de valores, a mensuração realizada através de números com suas respectivas unidades (Gatti, Witter \& Vitorino,2015).

Para a realização dos estudos In silico, as informações químicas como: estrutura química da molécula e massa molecular do Eugnol foram obtidas no programa Chemdraw (http://www.chemspider.com/). Com esses dados foi possivel realizar os estudos In silico proposto no trabalho.

O composto em estudo foi submetido aos softwares PASS (Prediction of activity spectra for substances) online e GUSAR, disponíveis de forma gratuita.

O PASS online é um software gratuito criado para avaliar o potencial biológico geral de uma molécula orgânica In silico sobre o organismo humano, ele permite previsões simultâneas de diversos tipos de atividades biológicas baseados na estrutura química dos compostos orgânicos. Além disso o software proporciona a estimativa do potencial de atividade de uma substância, podendo esta ser classificada como índices Pa (probabilidade "de ser ativo") e Pi (probabilidade "de ser inativo"), o mesmo pode ser acessado pelo endereço: (http://www.pharmaexpert.ru/passonline/) (SRINIVAS et al., 2014). Esta ferramenta computacional prevê com uma precisão de $95 \%$ o espectro de atividade de um composto químico específico, calculando de forma independente as duas probabilidades $(\mathrm{Pa}) \mathrm{e}(\mathrm{Pi})$.

O software GUSAR (http://www.pharmaexpert.ru/gusar/index.html) avalia a decomposição de estruturas químicas em descritores que são comparados com os descritores de compostos disponíveis em um banco de dados de compostos biologicamente ativos. Por meio desta ferramenta é possível realizar o estudo In silico para determinação da dose letal em 50\% (LD50) em ratos dos compostos químicos nas formas de administração subcutânea, intravenosa, via-oral e intraperitoneal (Lagunin, 2011).

\section{Resultados}

Os resultados do sistema operacional online PASS são expressados como um valor de probabilidade de ser ativo (Pa) e inativo (Pi). Para autenticar a atividade biológica dos compostos encontrados na amostragem, decidimos por usar apenas os valores de Pa acima de 0,700 (Parasuraman, 2011). A previsão das atividades antimutagênica do Eugenol (Figura 1) que apresentaram probabilidade de atividade (PA) maior que 0,700 (Tabela 1).

Figura 1. Estrutura química do Eugenol.

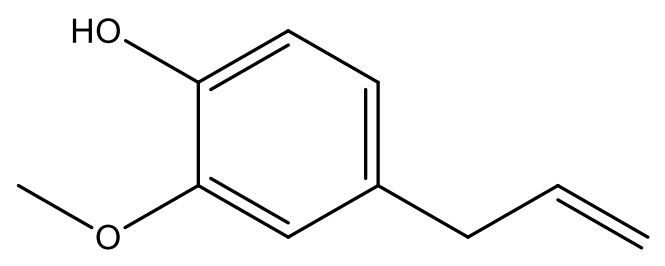

Fonte: Autores. 
Tabela 1. Potencial de atividade biológica com relação anticancerígenas previstas no PASS online.

\begin{tabular}{llc}
\hline $\mathbf{P a}$ & $\mathbf{P i}$ & Atividade \\
\hline 0,878 & 0,003 & Antimutagênico \\
0,873 & $\mathbf{0 , 0 0 4}$ & Estimulante Caspase 3 \\
0,803 & 0,004 & Tratamento pré-neoplásico \\
0,743 & 0,011 & Agonista da apoptose \\
\hline
\end{tabular}

Fonte: Autores.

$\mathrm{Na}$ tabela verifica-se que o Eugenol possui muitas propriedades que podem ser consideradas como promissores anticarcinogênicos. $\mathrm{O}$ mesmo apresentou um $\mathrm{Pa}>0,7$ para as atividades de Antimutagenese, estimulante de caspase 3, para o agonista de apoptose e favorável no tratamento pré neoplásico.

Ademais, para avaliação da toxicidade utilizou-se neste trabalho do software GUSAR. Pesquisando a DL50 do Eugenol pelo software, foi observado que na categoria 4, estão as vias de administração Intravenosa, oral, e subcutânea, indicando que é pouco tóxico. Já na categoria 5 enquadra-se a via intraperitoneal é relativamente atóxico. Como mostrado nas Tabelas 2, 3 .

Tabela 2. Dose letal (DL50) esperada para o Eugenol em diferentes vias de administração.

\begin{tabular}{cccc}
\hline $\begin{array}{c}\text { Administração } \\
\text { intraperitoneal }\end{array}$ & $\begin{array}{c}\text { Administração } \\
\text { intravenosa }\end{array}$ & $\begin{array}{c}\text { Administração oral } \\
\text { LD50 Log 10 (mg/ kg) }\end{array}$ & $\begin{array}{c}\text { Administração } \\
\text { subcutanea }\end{array}$ \\
LD50 Log 10 (mg/ kg) & $\begin{array}{c}\text { LD50 Log 10 (mg/ kg) } \\
\text { LD } \mathbf{~ ( m g / ~ k g ) ~}\end{array}$ \\
\hline $\mathbf{5 0 0 , 6}$ & 59,49 & 1083 & 717,3 \\
\hline
\end{tabular}

Fonte: Autores.

Tabela 3. Classificação aguda de toxicidade para roedores de produtos químicos pelo projeto OECD.

\begin{tabular}{cccc}
\hline $\begin{array}{c}\text { Administração } \\
\text { intraperitoneal }\end{array}$ & $\begin{array}{c}\text { Administração } \\
\text { intravenosa }\end{array}$ & Administração oral & $\begin{array}{c}\text { Administração } \\
\text { subcutanea }\end{array}$ \\
\hline Classe 5 & Classe 4 & Classe 4 & Classe 4 \\
\hline
\end{tabular}

Fonte: Autores.

\section{Discussão}

\section{Relacionados com Tratamento pré-neoplásico e ou quimiopreventivo}

Na pesquisa realizada por Min Ma et al, (2017), foi testado o uso de $1 \mathrm{mg}$ de eugenol em ratos possuindo células em fase pré-cancerígena de mama. Ela teve como resultado a possibilidade do Eugenol inibir significativamente o desenvolvimento de lesões pré-cancerosas da mama. Isso, através do bloqueio da sinalização HER2 / PI3K-AKT rede e a proliferação de células MCF-10AT positivas para HER-2. Tal achado resultou na apoptose da lesão pré-cancerosa da mama humana MCF-10AT (Ma, M.,2017). Já no trabalho de Moustafa Fathy, et al (2019), aponta que o efeito anticancerígeno propiciado pela substancia atua de maneira dose-dependente. 
A via de sinalização PI3K/AKT/mTOR quando alterada está amplamente presente em diversos processos neoplásicos, isso por exercer papeis importantes tais como a proliferação, síntese proteica, sobrevivência e a motilidade das células (Castaneda, Cortes, Gomez \& Ciruelos, 2010; Fathy et al, 2019; Ghayad, \& Cohen, P., 2010)

Os receptores da família HER (ErbB) são atuantes como receptores de fatores de crescimento com atividade tirosina cinase geralmente encontradas no processo de desenvolvimento, não encontrando em células adultas consideradas normais hematopoiéticas, apenas quando estão relacionadas a leucemias e linfomas (Freitas, 2008; Fruman \& Rommel,2014).

O efeito antimutagênico do Eugenol apresentou um $\mathrm{Pa}=0,878$. O estudo de Gökalp, Faik (2016) avaliou as estruturas geométricas e eletrônicas do Eugenol e concluiu que ao se dissolver no sangue ele pode interagir com radicais de outras moléculas, podendo retardar a formação de células cancerígenas ao impedir a deterioração aos radicais.

O estudo de Bezerra, Daniel et al (2017) também complementaram tal descoberta ao afirmar que os mecanismos atuantes contra as células cancerígenas estão na ação de prevenção por efeito antioxidante e, por efeito pró-oxidante que mata as células cancerígenas.

Já nos estudos de Jaganathan, Saravana Kumar et al (2011), foi observada diminuição da incidência e do tamanho dos tumores de pele, juntamente com o aumento geral na sobrevida dos ratos devido ao tratamento com eugenol, em experimentos com ratos contendo tumor de pele, sendo utilizados o eugenol por via oral 15 dias antes dos tratamentos quimioterápicos. Houve assim, a redução do estágio displásico juntamente com a taxa reduzida de proliferação celular e o aumento do apoptose, à regulação negativa da expressão de c-Myc, $\mathrm{H}$-ras e Bcl2, juntamente com a regulação positiva de P53, Bax e expressão ativa de Caspase-3 nas lesões cutâneas. Já o Bax é considerado um regulador do processo de apoptose, sendo ativado pela p53, e atua regulando a apoptose esta negativamente ou positivamente dependendo de sua proteína ligante (Chiu, Xue, Usuda Azizuddin \& Oleinick, 2003; Faria, Patrocínio, Filho \& Rabenhorst, 2006).

O gene p53 é ativado quando existe um dano celular, esse desempenha papel fundamental no desenvolvimento e falência celular. Essa tem um papel importantíssimo para prevenir a concentração de desregulações genéticas (Fett \& Salles, 2002; Mitani, 2001)

As vias de sinalização Ras/Raf/MEK/ERK podem atuar em diversos mecanismos de sobrevivência, migração e proliferação e por ativar várias vias de sinalização tais como a ERK1/2 e PI3K; sendo muito estudadas nos processos de tumorigênese (Meloche \& Pouysségur,2007; Toft \& Cryns,2011).

O gene bcl-2 é considerado anti-apoptótico, responsável por regular a ativação de caspases, e por isso é considerado favorável quando sua expressão é encontrada em carcinomas, como por exemplo o de mama (Derossi, Ito, Couto, José \& Bacchi, 2003; Facchini \& Penn,1998; Kelly, Cochran, Stiles \& Leder, 1983; Leve \& Morgado-Díaz,2012; Marcu, Bossone \& Patel, 1992; Scarff. \& Torloni,1968.)

No estudo de Arif Hussain et al (2011), foi avaliado o potencial quimiopreventivo do eugenol sozinho e em combinação com um agente quimioterapêutico como a gencitabina. Constataram que a análise da expressão de genes envolvidos no apoptose e inflamação revelou significativa regulação negativa de Bcl-2, COX-2 e IL-1ß no tratamento com eugenol.

Já nos estudos de Ali, Shakir et al (2014), relataram que o uso de Eugenol inibe a proliferação de células cancerígenas hepáticas e ao mesmo tempo diminui o estresse oxidativo, que pode ser o mecanismo de proteção contra a cirrose hepática prevenindo o câncer de fígado.

\section{Relacionados com tratamento de Neoplasias}

Eugenol tem propriedades anti-inflamatórias podendo ser utilizado para o tratamento do câncer de colo do útero (Hussain et al, 2011). Quando é usado para células cancerígenas esse é responsável por diminuir os tióis intracelulares 
em proteínas e faz uma função importante ao induzir a apoptose no HL60. Mostrando ser um potencial quimiopreventivo contra o câncer de cólon e na inibição do crescimento de células cancerígenas da próstata (Olayioye , Neve , Lane, Hynes ,2000).

A atividade antiproliferativa do eugenol é devido em sua estrutura ter um anel aromático. Esse, possui a capacidade de bloquear o câncer de colo do útero pelo efeito apoptótico de células HeLa, que apresentou forte atividade na concentração abaixo de 50 ppm, capaz de induzir células cancerígenas do colo do útero apoptóticas até 50\% (Rahman, Haykal, Siagian Sriepindonnta \& Tampubolon, 2018).

O Eugenol apresentou $\mathrm{Pa}=0,873$ para estimulação de caspase-3. De acordo com os estudos de Mingxia Jiang, et al (2020), um dos mecanismos de sobrevida das células tumorais está na desativação do processo de morte celular. Por isso, muitos estudos segundo o artigo concentram se nas pesquisas com a caspase 3, uma vez que esta é responsável por ativar tanto o processo de pioptose, quanto por apoptose. Ademais, segundo Huang, Q et al (2011), a caspase 3 também está inserida no processo de mediação do aumento no tamanho dos tumores sujeitos a tratamento radioterápico.

Segundo Liang, Wei-Zhe et al (2015) a interferência por via de caspases, também tem efeitos benéficos em células de glioblastoma humano. Averigou que essa substancia acarreta no aumento da produção de espécies reativas de oxigênio, e reduz o potencial da membrana mitocondrial, causando a liberação do citocromo c e na ativação das caspase-9 e a caspase-3, pode também induzir a via da apoptose mitocondrial.

Ademais no estudo de Ali, Shakir et al (2015) concluiu que o tratamento com Eugenol inibiu o crescimento e a proliferação de células humanas de câncer de mama MCF-7 através da indução de morte celular, sendo essa substancia dose dependente. No estudo demonstrou que as células tratadas com eugenol obteve um encolhimento celular, sangramento da membrana e a formação de corpo apoptótico. Além disso, o tratamento com eugenol também diminuiu o nível de glutationa intracelular e aumentou o nível de peroxidação lipídica.

No estudo realizado por Syed S. Islam e Abdelilah Aboussekhra (2019), feito In vitro, observou-se sobre a eficácia da combinação da cisplatina com o eugenol em duas células cancerígenas do ovário linhas SKOV3 e OV2774 usando as técnicas WST1 e citometria de fluxo, demonstrando que essa associação pode inibir sinergicamente tanto o crescimento, quanto a sobrevivência das células tumorais através da indução de apoptose.

\section{Efeitos tóxicos do Eugenol}

A literatura não há muitos artigos relatando sobre os efeitos tóxicos em humanos. Contudo, devido a este componente ser utilizado em larga escala como anestésico em animais (Gressler, Heinzmann, Baldisserotto, 2021), a maior parte de estudos envolvendo sobre sua toxicidade é relatada em peixes ou animais aquáticos.

Segundo Rothenstein et al (1983), este componente possui boa aceitação no uso tópico, sendo muito utilizado na indústria cosmética.

Na pesquisa de Goulet et al (2010) realizada com sapos africanos evidenciou danos renais e hepáticos dose e tempo dependentes ao serem expostos ao eugenol por 24 ou 72h. Contudo, após a interrupção da exposição esses danos foram reversíveis.

Nos estudos feitos por Grush et al. (2004) em Pseudoplatystoma corruscans jovens, ao introduzirem no aquário eugenol na dose de os peixes apresentaram hiperatividade, demonstrando comportamento de "tosse" com reflexo de regurgitação, tendo o L50 estimado de 96 horas em 21 ppm.

Já de acordo com os estudos de Martindale (1989) no ser humano a dose diária permitida para o eugenol é estimada em $2,5 \mathrm{mg} / \mathrm{kg}$. 
Ademais, VIDAL et al. (2008), avaliaram em Oreochromis niloticus que concentrações acima $81,97 \mathrm{mg} \mathrm{L}^{-1}$ foram tóxicos e $184,26 \mathrm{mg} \mathrm{L}^{-1}$ letais para esta espécie. Variando desta maneira a toxicidade e letalidade de acordo com cada organismo, seu tamanho, e composição estrutural e metabolismo.

\section{Conclusão}

O presente estudo demonstrou que o Eugenol possui elevado potencial de efeito antimutagênico. Ademais, vale ressaltar que pouco se sabe sobre sua toxicidade. Portanto, vê- se que necessitam de mais estudos e investimentos tecnólogos para a realização de ensaios pré clínicos com estudos In vivo e In vitro, com o objetivo de avaliar os efeitos biológicos mostrados no programa In Silico. Dessa forma, haverá uma melhor compreensão de seus efeitos, além do desfrute de seus benefícios com menores efeitos adversos.

\section{Referências}

Affonso, R. S., Rennó, M. N., Slana, G. B. C. A., \& Franca, T. C. C.(2012). Chemical and Biological Aspects of the Essential Oil of Indian Cloves. Revista Virtual de Química, 4(2), 146-161, 2012. http://dx.doi.org/10.5935/1984-6835.20120012.

Ali, S., Prasad, R. Mahmood, A., Routray, I., Shinkafi, T.S., Sahin, K., \& Kucuk, O. (2014). Eugenol-rich Fraction of Syzygium aromaticum (Clove) Reverses Biochemical and Histopathological Changes in Liver Cirrhosis and Inhibits Hepatic Cell Proliferation. Journal Of Cancer Prevention, 19 (4), 288-300. http://dx.doi.org/10.15430/jcp.2014.19.4.288.

Ali, S., Prasad, R., Mahmood, A., Routray, I., Shinkafi, T. S., Sahin, K., \& Kucuk, O. (2014). Eugenol-rich Fraction of Syzygium aromaticum (Clove) Reverses Biochemical and Histopathological Changes in Liver Cirrhosis and Inhibits Hepatic Cell Proliferation. Journal Of Cancer Prevention, 19 (4), 288300. http://dx.doi.org/10.15430/jcp.2014.19.4.288

Al-Sharif, I, Remmal, A, \& Aboussekhra, A. (2013). Eugenol triggers apoptosis in breast cancer cells through E2F1/survivin down-regulation. BioMed Central Cancer, 13:600. https://dx.doi.org/10.1186/1471-2407-13-600.

Bezerra, D., Militão, G., Morais, M. D., \& Sousa, D. D. (2017). The Dual Antioxidant/Prooxidant Effect of Eugenol and Its Action in Cancer Development and Treatment. Nutrients, 9(12), 1367-1382. http://dx.doi.org/10.3390/nu9121367.

Castaneda, C., A., Cortes, H. F., Gomez, H. L., \& Ciruelos, E. M. (2010). The phosphatidyl inositol 3- kinase/AKT signaling pathway in breast cancer. Cancer Metastasis, v. 29, 751-759. https://doi.org/10.1007/s10555-010-9261-0.

Chiu, S. M, Xue L.Y., Usuda, J, Azizuddin K., \& Oleinick, N. L. (2003). Bax is essential for mitochondrion-mediated apoptosis but not for cell death caused by photodynamic therapy. British Journal Of Cancer, 1590-1597. 10.1038/sj.bjc.6601298.

Danchin A., Medigue C., Gascuel O., Soldano H., \& Henaut A. (1991). From data banks to data bases. Research in Microbiology, 142 (7-8), 913-6. Http://dx.doi.org/10.1016/0923-2508(91)90073-J

Derossi, D. R., Ito, K., Couto, F., José D. O., \& Bacchi, C. E. (2003). Avaliação da expressão da proteína bcl-2 no carcinoma de mama: estudo em punção aspirativa por agulha fina, correlação com grau histológico em espécimes cirúrgicos correspondentes. Jornal Brasileiro de Patologia e Medicina Laboratorial, 39(3), 229-235. Http://dx.doi.org/10.1590/s1676-24442003000300010

Facchini, L. M., \& Penn, L. Z. (1998). The molecular role of Myc in growth and transformation: recent discoveries lead to new insights. FASEB Journal, $12(1), 633-51$.

Faria, M. H. G, Patrocínio, R. M. S. V. Filho, M. O. M., \& Rabenhorst, S. H. (2006). Expressão das proteínas BCL-2 e BAX em tumores astrocíticos humanos. Jornal Brasileiro de Patologia e Medicina Laboratorial, 42(4), 271-278. https://doi.org/10.1590/S1676-24442006000400008.

Fathy, M., Fawzy, M. A., Hintzsche, H., Nikaido, T., Dandekar, T., \& Othman, E. M. (2019) Eugenol Exerts Apoptotic Effect and Modulates the Sensitivity of HeLa Cells to Cisplatin and Radiation. Molecule, 24(21), 3979-3393. http://dx.doi.org/10.3390/molecules24213979.

Fett, C., Agnes C., \& Salles, A. B. C. F. (2002). A importância do gene p53 na carcinogênese humana. Revista Brasileira Hematologia Hemoterapia, 24(2), 85-89. http://dx.doi.org/10.1590/S1516-84842002000200004.

Filimonov, D. A, Lagunin, A.A, Gloriozova T. A., Rudik A. V., Druzhilovskii D. S., Pogodin, P. V., \& Poroikov V. V. (2014). Prediction of the biological activity spectra of organic compounds using the PASS online web resource. Chemistry of Heterocyclic Compounds. 50 (3), 444457.https://doi.org/10.1007/s10593-014-1496-1

Freitas, C. S. (2008). Estendendo o Conhecimento sobre a Família Her-Receptores para o Fator de Crescimento Epidérmico e seus ligantes às Malignidades Hematológicas. Revista Brasileira de Cancerologia, 1(54), 79-86. www1.inca.gov.br/rbc/n_54/v01/pdf/revisao_5_pag_79a86.pdf.

Fruman, D. A., \& Rommel, C. (2014). PI3K and cancer: lessons, challenges and opportunities. Nature Reviews Drug Discovery, 13(2), 140-156. Http://dx.doi.org/10.1038/nrd4204. 
Gatti, A. L., Witter, C., Gil, C. A., \& Vitorino, S. D. S. (2015). Psychologically focused group intervention with the elderly: A qualitative research.Psicologia: Ciência e Profissão, 35(1), 20-39.

Ghayad, S., \& Cohen, P. (2010). Inhibitors Of The PI3K/Akt/Mtor Pathway: new hope for breast cancer patients. Recent Patents On Anti-Cancer Drug Discovery. Bentham Science Publishers Ltd, 5(1), 29-57. http://dx.doi.org/10.2174/157489210789702208.

Gökalp, F. (2006). A Study On The Chemical Properties Of Eugenol And Eugenol Acetate, Clove Essential Oils. Sigma Journal Engineering And Natural Sciences Sigma, 3(34), 407-414.

Goulet, F., Hélie, P., \& Vachon, P. (2010). Eugenol anesthesia in African clawed frogs (Xenopus laevis) of different body weights. Journal of the American Association for Laboratory Animal Science: JAALAS, 49(4), 460-463.

Gressler, L. T., Heinzmann, B. M., \& Baldisserotto, B. (2021). Analgesia, anesthesia, and euthanasia of aquatic animals. Aquaculture Pharmacology, [S.L.], Elsevier, p. 297-346. Elsevier. http://dx.doi.org/10.1016/b978-0-12-821339-1.00007-6.

Grush, J. , Noakes, D. L. G. , \& Moccia, R. D. (2004).The efficacy of clove oil as an anesthetic for the zebrafish, Danio rerio (Hamilton). Zebrafish,1(1), 4653. https://doi.org/10.1089/154585404774101671

Huang, Q., Li, F., Liu, X., Li, W., Shi, W., Liu, F. F., O'Sullivan, B., He, Z., Peng, Y., Tan, A. C., Zhou, L., Shen, J., Han, G., Wang, X. J., Thorburn, J., Thorburn, A., Jimeno, A., Raben, D., Bedford, J. S., \& Li, C. Y. (2011). Caspase 3-mediated stimulation of tumor cell repopulation during cancer radiotherapy. Nature medicine, 17(7), 860-866. https://doi.org/10.1038/nm.2385

Hussain, A., Brahmbhatt, K., Priyani, A., Ahmed, M., Rizvi, T. A., \& Sharma, C. (2011). Eugenol Enhances the Chemotherapeutic Potential of Gemcitabine and Induces Anticarcinogenic and Anti-inflammatory Activity in Human Cervical Cancer Cells. Cancer Biotherapy And Radiopharmaceuticals, 26(5),519527. http://dx.doi.org/10.1089/cbr.2010.0925

Hussain, A., Brahmbhatt, K. Priyani, A., Ahmed, M., Rizvi, T. A., \& Sharma, C. (2011). Eugenol Enhances the Chemotherapeutic Potential of Gemcitabine and Induces Anticarcinogenic and Anti-inflammatory Activity in Human Cervical Cancer Cells. Cancer Biotherapy And Radiopharmaceuticals, 26(5),519527. http://dx.doi.org/10.1089/cbr.2010.0925.

Islam, S. S., \& Aboussekhra, A. (2019). Sequential combination of cisplatin with eugenol targets ovarian cancer stem cells through the Notch-Hes1 signalling pathway__Journal of Experimental \& Clinical Cancer Research, 38(382),1-14. http://dx.doi.org/10.1186/s13046-019-1360-3

Jaganathan, S. K., Mondhe, D., Wani, Z. A., Pal, H. C., \& Mandal, M. (2010). Effect of Honey and Eugenol on Ehrlich Ascites and Solid Carcinoma. Journal Of Biomedicine And Biotechnology1-5. http://dx.doi.org/10.1155/2010/989163.

Jiang, M., Qi, L., Li, L. \& Li, Y. (2020). The caspase-3/GSDME signal pathway as a switch between apoptosis and pyroptosis in cancer. Cell Death Discovery. 6 (112), 2-11. https://doi.org/10.1038/s41420-020-00349-0

Kelly, K., Cochran, B. H., Stiles, C. D., \& Leder, P. (1983). Cell-specific regulation of the c-myc gene by lymphocyte mitogens and platelet-derived growth fator.Cell, 35(1), 603-10.

Lagunin, A., Zakharov, A., Filimonov, D., \& Poroikov, V. (2011). QSAR Modelling of Rat Acute Toxicity on the Basis of PASS Prediction. Molecular informatics, 30(2-3), 241-250. https://doi.org/10.1002/minf.201000151.

Leve, F., Morgado-Díaz, J. A. (2012). Rho GTPase signaling in the develop- ment of colorectal cancer. Journal of Cellular Biochemistry, 113(1), 2549-2559. https://doi.org/10.1002/jcb.24153

Liang, W. Z., Chou, C. T., Hsu, S. S., Liao, W. C., Shieh, P., Kuo, D. H., Tseng, H. W., Kuo, C. C., Jan, C. R. (2015). The involvement of mitochondrial apoptotic pathway in eugenol-induced cell death in human glioblastoma cells. Toxicology Letters, 232(1),122-132. http://dx.doi.org/10.1016/j.toxlet.2014.10.023.

Lutfi, M. , \& Roque, N. F. (2014). Eugenias Histories. Química Nova na Escola, 36 (4),252-260. Http://dx.doi.org/10.5935/0104-8899.20140030.

Ma, M., Ma, Y., Zhang, G., Liao, R., Jiang, X., \& Yan, X. (2017). Eugenol alleviated breast precancerous lesions through HER2 / PI3K-AKT pathwayinduced cell apoptosis and S-phase arrest. Oncotarget, 8(34), 56296-310. Https://doi.org/10.18632/oncotarget.17626.

Manikandan, P., Murugan, R. S., Priyadarsini, R. V., Vinothini, G., \& Nagini, S. (2010). Eugenol induces apoptosis and inhibits invasion and angiogenesis in a rat model of gastric carcinogenesis induced by MNNG. Life sciences, 86(25-26), 936-941. https://doi.org/10.1016/j.lfs.2010.04.010

Marcu, K. B., Bossone, S. A., \& Patel, A. J. (1992). Myc function and regulation. Annual Review Biochemisty, 61(1), 806-60.

Meloche,S., \& Pouysségur, J.(2007). “TheERK1/2mitogen-activatedproteinki- nase pathway as a máster regulator of the G1- to S-phase transition”. Oncogene, 26(1), 3227-3239. http://dx.doi.org/10.1038/sj.onc.1210414.

Mitani, K. (2001). Disease-related gene and tumor progression. Nippon Rinsho, 59(1), 2316- 2321.

NTP-National Toxicology Program, Institute of Environmental Health Sciences, National Institutes of Health (NTP). (1992).Le National Toxicology Program Chemical Repository Database. Research Triangle Park, North Carolina.

Olayioye, M. A, Neve, R. M, Lane, H. A, \& Hynes, N. E. (2000) .The ErbB signaling network: receptor heterodimerization in development and cancer. EMBO Jornal, 19(1), 3159-67. Http://dx.doi.org/10.1093/emboj/19.13.3159.

Palsson, B. (2000). The challenges of in silico biology. Nature Biotechnology, 18 (1), 1147-1150. Http://dx.doi.org/10.1038/81125. 
Research, Society and Development, v. 10, n. 4, e27910414165, 2021

(CC BY 4.0) | ISSN 2525-3409 | DOI: http://dx.doi.org/10.33448/rsd-v10i4.14165

Paoli, S., Giani, S. T., Presta, S. P., Giani, T. S., Presta, G. A., Marcia Oliveira Pereira, M. O., Fonseca, A. S., Neto, J. B., Medeiros, A. C., Filho, S. D. S., \&Filho, M. B.(2007). Effects of clove (Caryophyllus aromaticus L.) on the labeling of blood constituents with technetium-99m and on the morphology of red blood cells. Brazilian Archives of Biology and Technology, 50(spe), 175-182. https://dx.doi.org/10.1590/S1516-89132007000600022.

Parasuraman, S. (2011).Toxicological screening. Journal of Pharmacology \& Pharmacotherapeutics , 2(2),74-79. Https://doi.org/ 10.4103/0976-500X.81895.

Rahman, M. F. , Haykal, M. N. , Siagian, N. A. Sriepindonnta, P. M. , \& Tampubolon, N. A. (2018). Synthesis and Proapoptotic Activity on Cervical Cancer Cell of Ester Eugenol 1-(3-Methoxy-4-hydroxy)phenyl-2-propylmethanoate. Iop Conference Series: Materials Science and Engineering, 299(1),1-7. http://dx.doi.org/10.1088/1757-899x/299/1/012071.

Roque, M. L. N. F.(2014). Histórias de Eugênias. Química e Sociedade, 36 (1), 252-260.Http://dx.doi.org/10.5935/0104-889920140030.

Rothenstein, A. S., Booman, K. A., Dorsky, J., Kohrman, K. A., Schwoeppe, E. A., \& Sedlak, R. I. (1983). Steltenkamp. Eugenol and clove leaf oil. A survey of consumer patchtest sensitization. Food and Chemical Toxicology, 21(1), 727-733. http://dx.doi.org/10.1016/0278-6915(83)90204-1

Ruttkies, C., Schymanski, E. L., Wolf, S., Hollender, J, \& Steffen, N. (2016). MetFrag relaunched: incorporating strategies beyond in silico fragmentation. Journal of Cheminformatic, 8(3) .Https://doi.org/10.1186/s13321-016-0115-9

Scarff, R. \& Torloni, F. (1968). Histological typing of breast tumors. International histological classification of tumors. Geneva: World Health Organization. http://dx.doi.org/10.1093/ajcp/78.6.806

Scherer, R., Wagner, R., Duarte, M. C. T., \& Godoy, H. T. (2009). Composição e atividades antioxidante e antimicrobiana dos óleos essenciais de cravo-daíndia, citronela e palmarosa. Revista brasileira plantas medicinais, 11(.4), 442-449. Http://dx.doi.org/10.1590/S1516-05722009000400013

Sotomayor, M., \& Schulten, K. (2007). Single-Molecule Experiments in Vitro and in Silico. American Association for the Advancement of Science (AAAS). Science, 316 (582), 1144-1148. http://dx.doi.org/10.1126/science.1137591.

Srinivas, N., Sandeep, K. S., Anusha, Y., \& Devendra, B. N. (2014). In Vitro Cytotoxic Evaluation and Detoxification of Monocrotaline (Mct) Alkaloid: An In Silico Approach. Journal of Biochemistry and Bioinformatics, 2(3).20-29.

Toft D. J., \& Cryns V. L. (2011). Minireview: Basal-Like Breast Cancer: From Molecular Profiles to Targeted Therapies. Molecular Endocrinology, 25(2), 199- 211. http://dx.doi.org/ 10.1210/me.2010-0164.

Wingate D. (1989). Martindale: The extra pharmacopeia (29th ed). Gut, 30(12), 1804.Vidal, Lvo.(2008).Eugenol como anestésico para tilápia do Nilo. Pesquisa Agropecuária Brasileira, 43(8), 1069-1074. http://dx.doi.org/10.1590/S0100-204X2008000800017 